\title{
Medicamentos innovadores: hacia un modelo colaborativo
}

\author{
Eduardo Satué de Velasco \\ Presidente de SEFAC Aragón, La Rioja y Navarra.
}

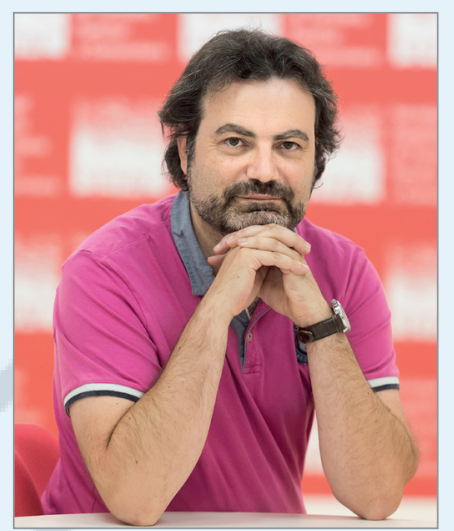

Eduardo Satué de Velasco
Este año se cumple una década en la que varias autonomías comenzaron a trasladar medicamentos de Diagnóstico Hospitalario (DH) de las farmacias comunitarias $(\mathrm{FC})$ a la farmacia hospitalaria $(\mathrm{FH})$. Independientemente de que las autonomías carecían (y carecen) de competencias para realizar este cambio, la realidad es que casi toda la innovación en medicamentos que sale en nuestro país está dejando al margen a la farmacia comunitaria y acaba en la farmacia hospitalaria y esta tendencia no tiene intención de cambiar gracias a los inefables DIHSC, medicamentos de "Diagnóstico Hospitalario Sin Cupón precinto", cajón de sastre que en la práctica tiene como principal criterio recoger determinados medicamentos de precio elevado. Los farmacéuticos comunitarios nos hemos movido al respecto esta década entre el dejar hacer, la queja y la ingenuidad de pensar que nuestra accesibilidad es argumento más que suficiente para que vuelvan sin más. Heráclito ya explicaba hace más de dos mil años que uno no se puede bañar dos veces en el mismo río, lo que traducido a nuestra situación significa que los famosos medicamentos DIHSC no llevan intención de volver a la FC en la misma situación que hace una década. Y esto será así por varios motivos: el primero es el económico por partida doble. Ni la administración va a querer dejar de tener la sensación de control o dicho de otro modo, la posibilidad de negociar descuentos que ahora consigue (aunque haya una total opacidad al respecto y ni ella misma sepa si ahorra) ni el modelo retributivo actual de estos medicamentos en FC consistente en una tarifa plana y baja le permite a esta asumir costes muy elevados. El segundo motivo y casi tan importante es que los años pasan y la farmacia hospitalaria ya ha cogido carrerilla al respecto. Este grupo de fármacos les está dando una visibilidad y un cierto poder con el que no contaban antes y no lo van a dejar perder así como así. Aunque los resultados sean dispares, la FH se está esforzando por aportar valor aña- dido en la dispensación de estos fármacos que difícilmente puede equipararse al que aportaría la farmacia comunitaria. Así, para que estos medicamentos se dispensen en su entorno natural que es la farmacia comunitaria debemos hacer valer nuestras fortalezas. Está claro que la accesibilidad es una de las principales, pero no la única. La longitudinalidad (atención continuada en el tiempo), el conocimiento global de los tratamientos del paciente (no solo los de prescripción sino también la automedicación, la fitoterapia, los complementos nutricionales...), la alta frecuencia de visitas de los pacientes, la coordinación con el médico de atención primaria... son activos muy valiosos a los que se les debería sacar más partido con una capacitación específica para este tipo de fármacos y estructurados en torno a protocolos de trabajo (como seguimiento, conciliación, revisión de uso, SPD...), zonas de atención personalizada y coordinados con el resto de profesionales sanitarios. Todo esto permitiría que la farmacia comunitaria siga siendo lo que nunca debe dejar de ser, el punto de acceso y seguimiento de medicamentos para el paciente ambulatorio.

Sin embargo, por bueno que pueda ser este modelo de farmacia comunitaria para la dispensación de este tipo de medicamentos, el modelo de farmacia del siglo XXI no puede seguir obligando al paciente a tener que dividirse entre las dos farmacias, cada una viviendo de espaldas a la otra ya que esto no puede más que alimentar problemas con los medicamentos. Los avances consistentes en la medicina personalizada, toda la casuística que se va a derivar de un despliegue de posibilidades farmacodinámicas y farmacocinéticas hacen imposible que sólo la FH o la FC puedan hacerse cargo por sí solos y más importante, el paciente no es de nadie sino de él mismo y tiene derecho a que los celos profesionales no afecten a que el tratamiento que reciba sea lo mejor posible. La atención al paciente exige, y ya tardamos, una coordinación y una integración en el trabajo diario con estos fármacos. 
Es preciso adentrarse en la complejidad de los distintos pasos que implican estos tratamientos como diagnóstico, adquisición, preparación, dispensación y seguimiento de los medicamentos y dejar de asumirlos como un paquete global sino situar cada uno donde mejor pueda servir a la población y recapitalizar científicamente la atención primaria. Tal como SEFAC lo ve, la farmacia hospitalaria equivocaría su papel si pretende convertirse en una farmacia comunitaria de medicamentos de élite, esto es, atender masivamente a la población que use estos fármacos obviando que ni dispone ni puede disponer de una estructura adaptada para este fin. Por su parte, la farmacia comunitaria se equivocaría si cree que puede prestar el mismo afine del que dispone un hospital y su capacidad de generar pruebas, perfiles farmacocinéticos en los momentos de inicio o de cambios de medicación... El modelo de farmacia colaborativa del siglo xxi debe ser capaz de complementar los puntos fuertes de cada una al servicio del paciente. En primer lugar es preciso realizar una revisión de qué medicamentos deben ser hospitalarios $(\mathrm{H})$, de diagnóstico hospitalario (DH), de especial control médico (ECM)... en función de criterios únicamente científicos y no meramente económicos (por ejemplo, la mayoría de antirretrovirales podrían considerarse DH actualmente). En aquellos medicamentos DH o ECM que se considere que puedan requerir medidas de seguridad adicionales deben establecerse protocolos que indiquen las condiciones especiales de dispensación, qué circuito debe establecer el paciente entre FC y FH, cuáles serían los protocolos específicos para cada medicamento que integraran a los diferentes profesionales implicados, incluyendo episodios de vuelta al hospital en caso de alertas o necesidades especificadas y, en definitiva, generando un circulo sinérgico de colaboración. En el modelo actual de adquisición de muchos de los medicamentos innovadores por parte de la farmacia hospitalaria, la distribución farmacéutica está más que preparada para realizar el circuito de estos medicamentos entre FH y FC y sería más que razonable pensar que se puede llegar a un acuerdo de pago por servicio de estas dispensaciones y los procesos adicionales que se precisen de la FC.

Para generar este modelo es preciso que las sociedades científicas, los colegios, las instituciones... nos pongamos de acuerdo pero también es importante que quien tiene la responsabilidad de generar el mejor servicio posible al ciudadano, la Administración, lidere y sepa optimizar los recursos disponibles ya existentes. Como profesión, y me refiero a todo el colectivo, estamos obligados a buscar el mejor modelo, que equilibre lo que ya funciona con lo que todavía debe crearse porque si no lo hacemos así, no estaremos cumpliendo la misión que la sociedad nos encomendó cuando nos otorgó el título de farmacéuticos, independientemente de donde estemos trabajando. 